\title{
Les archives de presse
}

\section{Dominique Cotte}

\author{
Université de Lille-3 \\ UFR IDIST \\ $B P 159$ \\ F-59659 Villeneuve d'Ascq Cedex \\ cotte@univ-lille3.fr
}

\begin{abstract}
RÉSUMÉ : La notion "d'archives » revêt une dimension particulière dans les entreprises de presse. Au-delà de la mémoire interne, les services de documentation gèrent également des sources externes. Cet article retrace les principales étapes, à la fois organisationnelles et techniques, qui ont rythmé les trois dernières décennies. La dernière en date, le passage aux langages de balisage, notamment XML, risque d'introduire cette fois-ci une véritable rupture. ABSTRACT: The "archive " have a special dimension in media industry. The libraries do not process only an internal memory, but also external resources. This article reminds the main steps, both organisational and technical, that occured in the last three decades. The last one, tagging languages, especially XML, may introduce this time a real difference.
\end{abstract}

MOTS-CLÉS : archives, documentation, presse, gestion électronique de documents.

KEY WORDS: archive, library, press, electronic document storage.

Document numérique. Volume $4-n^{\circ} 3-4 / 2000$, pages 331 à 342 


\section{Introduction}

Dans l'expression « archives de presse », on pourra noter plusieurs connotations différentes. En filigrane, toutes font appel à la reconnaissance du rôle fondamental de la presse comme mémoire de la société. Les «archives de presse » sont considérées comme un patrimoine, l'outil du journaliste, mais aussi du chercheur, du politique, du détective, et du citoyen lambda. Cependant, la matière que contiennent ces archives particulières, l'information, en font une catégorie à part. La notion de péremption par exemple, est beaucoup plus difficile à cerner, précisément parce qu'un sujet peut revenir brutalement dans l'actualité des dizaines d'années après. Comme toute entreprise en tant qu'unité juridique, les journaux et magazines, chaînes de télévision et de radio, possèdent leurs archives internes : comptabilité, contrats, documents administratifs, etc. Ce type d'archives ne se distingue a priori pas fondamentalement de ce qui existe dans d'autres secteurs professionnels, et nous ne l'aborderons pas ici. En revanche, ce qui forme le produit même du journal, sa matière première, constitue une catégorie spécifique d'archives.

\section{Des archives particulières}

On ne trouvera sans doute plus guère aujourd'hui de titres dans lesquels on emploie encore le mot «archives » pour désigner le service chargé de conserver la mémoire du journal. Ici comme ailleurs, le terme est chargé d'un sens vieillot, péjoratif, immobiliste. Pour peu que le service en question soit éloigné physiquement des centres vitaux du journal (et notamment de la rédaction), voire relégué dans des sous-sols, on aura tout de l'image repoussoir des « archives ». La plupart des services d'archives des organismes de presse portent actuellement le nom de «service de documentation ${ }^{1} »$. La querelle n'est pas que sémantique bien entendu, elle fait appel à des représentations complexes et à une subtile répartition des hiérarchies sociales dans l'entreprise; mais surtout elle est révélatrice d'une nature particulière des archives de presse, et des fonctions que sont appelés à remplir ceux qui les gèrent, les documentalistes de presse.

Si nous voulions nous conformer à une définition stricte, il faudrait dire que ne sont considérés comme des « archives de presse » que les articles issus des numéros du journal parus au fil du temps. Or, la nature du travail journalistique est telle que, la plupart des temps, cette source unique d'information rétrospective est insuffisante et que les services de documentation font un travail de sélection, dépouillement et

1. Mais, si on ne parle plus guère «des archives» pour désigner, en interne le service de documentation, le vocable réapparaît sur les sites web de certains titres. Par exemple les sites web du Monde, des Echos, de La Tribune, possèdent des onglets ou des rubriques intitulés « archives » et renvoyant, en fait vers les moteurs de recherche internes. 
conservation de nombreux autres titres que les leurs propres ${ }^{2}$. Physiquement, ces documents forment un vaste corpus dans lequel, la plupart du temps, se trouvent regroupés à la fois les articles du titre lui-même et les articles d'autres journaux. On touche là à la différence de pratique entre un service de documentation et un service qui ne serait dédié qu'à la conservation des archives proprement dites du titre. Les politiques suivies dépendent ici à la fois de facteurs culturels et de la différence de moyens affectés par les patrons de presse à cette activité. Si le Monde, journal de référence, a longtemps vécu en ne constituant son fonds que des articles du Monde, à l'inverse l'Express, sous l'impulsion de «l'américanisme» de son fondateur, Jean-Jacques Servan-Schreiber adapte le modèle américain de la library qui dépouille de nombreuses sources extérieures au journal. Ainsi, dans le service de documentation, la fonction «archives » qui a pour mission de conserver la mémoire interne, et la fonction « documentation », qui vise à constituer une mémoire externe, se confondent.

\section{Mémoire interne et mémoire externe}

En bonne logique, seule la mémoire interne, composée des articles parus dans le journal concerné, devrait être qualifiée «d'archives». Mais il se trouve que l'on attribue au même service («les archives» ou plus généralement aujourd'hui «la documentation ») la gestion des deux types de ressources, la mémoire externe étant composée d'extraits de presse des journaux concurrents ou d'autres titres. Si l'on se place du point de vue de l'utilisateur, c'est-à-dire du journaliste, l'approche est une approche thématique : conflit bosniaque, vache folle, coupe du monde de football, parité en politique, délinquance, etc. sont les clés d'entrée d'une recherche documentaire pour répondre aux questions des journalistes, et ceci quel que soit le moyen documentaire utilisé pour satisfaire cette requête : dossiers documentaires, banques de données professionnelles, sites web, etc. De ce point de vue-là, les archives de presse forment bien un tout, organisé selon une classification par sujets, dans le but de pouvoir reconstruire la mémoire d'un événement en cas de besoin. Très rares sont aujourd'hui les titres de presse qui pourraient prétendre vivre sur l'archivage de leur seule matière produite, sans l'associer, et donc la croiser avec d'autres ressources émanant d'autres titres.

En revanche, du point de vue des modes de traitement, l'origine de l'information n'est pas neutre, et la distinction interne/externe constitue encore un point de démarcation important.

Le traitement des sources internes a subi d'importantes modifications, dès lors que la production des journaux a été entièrement informatisée. Pierre Albert [ALB 84] relève que l'existence d'index ou de tables est quasiment contemporaine

2. Plus de 200 pour le service de documentation de Libération, plus de 300 pour celui de l'Express, par exemple. 
de la presse moderne. L'approche reste ici bibliographique et référentielle et permet d'organiser une mémoire de l'information que la consultation de la collection seule rendrait vite fastidieux. Des journaux comme Ouest-France ou le Monde ont jusqu'à l'informatisation, utilisé des fichiers de type bibliographique pour décrire au jour le jour le contenu de leurs publications. Mais comme tout dispositif référentiel, celui-ci suppose la dissociation physique du support de description et de la chose décrite. Sans collection du journal, pas de possibilité d'accéder à l'article par sa référence, et donc à l'information. La diffusion des archives internes reste donc en tout état de cause limitée, et elle est souvent couplée avec une activité de réponse aux lecteurs, ou pour les titres les mieux équipés, fait l'objet d'un service de consultation à heure fixe, pour le grand public.

Il est clair que l'informatisation progressive de la chaîne de production des journaux va considérablement élargir ces possibilités de diffusion, à compter du début des années 1970. Le passage des techniques traditionnelles de composition au plomb à la photocomposition et l'augmentation des capacités des mémoires de stockage informatiques a permis de créer des archives électroniques sous la forme de banques de données interrogeables en texte intégral. L'ouverture des archives électroniques du New York Times en 1971 marque de ce point de vue une rupture dans les modes de stockage de l'information. Il est désormais possible, non seulement d'associer la description documentaire (champ Descripteurs) à l'objet décrit, mais également de permettre la consultation de celui-ci à distance. De grands serveurs comme Dialog et surtout Lexis-Nexis se font une spécialité de l'hébergement de banques de données de presse en texte intégral, notamment américaines, la France restant relativement en retard dans ce domaine pendant au moins quinze ans (la première banque de données de presse en texte intégral française disponible sur serveur, celle de l'AFP, date de 1984, la deuxième, celle du Monde, date de 1987).

Tout au long de cette époque, les serveurs deviennent, d'une certaine manière, des super centres d'archives électroniques permettant, grâce à des regroupements thématiques par base ou à la pratique des clusters, de faire des recherches transversales dans plusieurs titres. Cependant, dans les centres de documentation de presse, la pratique de l'utilisation des banques de données sur serveur, même si elle se répand à partir de la fin des années 1980, ne remplace jamais la sélection et l'archivage des sources papier. Les raisons en sont multiples :

- la documentation de presse est très attachée à la logique du dossier thématique. C'est la manière la plus synthétique de rassembler l'information, et surtout de l'organiser pour pouvoir répondre très rapidement aux demandes d'information sur 
les sujets les plus variés; or, la logique du texte intégral ${ }^{3}$ est beaucoup mieux adaptée aux recherches de type analytique ;

- la faiblesse de l'offre, notamment française, a longtemps empêché tout effet de substitution entre les sources papier et les sources électroniques ;

- les journalistes, attachés à la forme papier, à la hiérarchie visuelle de la mise en page et à la contextualisation de l'information sont déroutés par «l'effet listing » produit lors de l'édition de résultats d'interrogation de banques de données ;

- les coûts d'interrogation, compte tenu du besoin important en matière d'archivage de presse, sont trop élevés.

Par conséquent, même si les journaux deviennent producteurs d'archives électroniques, ils continuent à archiver leur propre production sous forme papier, et surtout à collecter la matière externe formée des différents journaux dépouillés par le centre de documentation.

Car le paradoxe est bien là : chaque journal dispose de ses archives électroniques, et les met par le truchement des serveurs à la disposition du public, mais pour accéder à la matière des autres titres il lui faut continuer à traiter du papier.

Le traitement des sources externes a lui aussi évolué depuis une vingtaine d'années, notamment avec l'apparition des supports de stockage optiques dans les années 1980. Comme nous l'avons vu, la matière documentaire composée d'articles de journaux différents est indispensable à l'exercice de la mission du journaliste. L'accès à des dossiers thématiques permet :

- de s'informer sur un thème peu connu ;

- de contextualiser un événement ;

- de restaurer le fil historique d'un sujet ;

- de comparer les modes de traitement journalistiques selon les titres ;

- de trouver des idées de sujets ou d'enquêtes ;

- d'alerter sur des problématiques nouvelles.

La plupart des grands centres de documentation de presse dépouillent ainsi plusieurs dizaines voire plusieurs centaines de titres et intègrent annuellement plusieurs centaines de milliers de pages à leur fonds documentaire. Cette pratique

3. Le bruit créé par le texte intégral est important. Certains centres de documentation de presse, comme celui de Libération par exemple, ont inauguré récemment une nouvelle étape avec le passage à des outils d'indexation semi-automatique empruntant au traitement automatique du langage. 
pose évidemment des problèmes d'archivage complexes ${ }^{4}$. Les pratiques et les outils sont de configurations variables. Sur le plan matériel, toutes les solutions sont mises en œuvre, depuis le dossier suspendu jusqu'aux boîtes d'archives, en passant par les tiroirs. Sur le plan documentaire, différents types de plans de classement ${ }^{5}$ sont utilisés, de type alphabétique ou alpha-numérique.

Le temps de collecte et de traitement matériel (découpage, collage, classement), est évidemment considérable, mais c'est la condition sine qua non pour pouvoir localiser précisément l'information dans des délais extrêmement brefs (quelques minutes dans la presse quotidienne), délais qui constituent une des caractéristiques du travail documentaire dans la presse. Il n'empêche que ce travail, parfaitement utile en soi, semble souvent - et particulièrement aux utilisateurs - dévalorisé et effectué au détriment des tâches plus «nobles ${ }^{6}$ que sont la recherche et la réponse aux questions. Par conséquent, le recours aux Nouvelles technologies de l'information a visé autant la résolution des problèmes physiques de stockage que l'automatisation de ces tâches.

On notera, avant d'aborder ces sujets, que les systèmes anglo-saxons, et notamment américains ont toujours développé, contrairement à la logique à l'œuvre en France, des pratiques classiques d'archivage, notamment en codifiant le traitement à apporter dans le domaine de la conservation des documents. Le New York Times, par exemple, dispose pour sa library d'une charte précisant la durée de conservation des documents en fonction du titre dont ils sont issus. Cette hiérarchie fournit une solution commode et rationnelle pour éviter de se laisser déborder par la marée de papier. L'esprit français craint, en utilisant de telles méthodes, de laisser échapper des contenus précieux. A l'approche par l'autorité (basée sur la notoriété des titres de presse et leur hiérarchie), on substitue ici une approche par les contenus.

Dans les années 1970, une des solutions envisagées pour gérer les problèmes d'occupation de l'espace, se base sur la technologie des microformes : microfilms pour les collections de journaux, microfiches pour les dossiers documentaires. Dans

\footnotetext{
4. Notons que cette pratique, concernant des sources extérieures, n'est pas spécifique à la presse. De nombreuses administrations ou entreprises qui utilisent la presse comme source documentaire créent des dispositifs comparables à ceux existant dans les documentations de presse. La banque Paribas a, par exemple, longtemps constitué une référence en matière de GED appliquée à des documents de presse, pour le service de documentation de son département Etudes.

5. La plupart des systèmes manuels disposent de plans de classement thématiques ou alphabétiques, l'apparition de thesaurus est contemporaine de la création des premières banques de données internes.

6. Pour l'opposition entre «noble » et « vil » dans le domaine du traitement de l'information, voir Béatrice Vacher, La gestion de l'information en entreprises, Enquête sur la ruse, l'étourderie, l'oubli et le bricolage organisés, ADBS, 1997.
} 
certains cas, l'existence de ces supports a donné lieu à une première tentative d'informatisation (dans le groupe Hachette - Edi7 à l'époque - par exemple) qui associent la description logique des documents dans une base de données documentaires et le classement mécanique des fiches dans des caroussels, ou la lecture automatique des microfilms au moyen de pavés numériques. Cependant, les microformes ne constituent qu'un pis-aller car leur consultation est relativement lente. Déjà cette première forme d'informatisation soulève un problème redoutable que les technologies ultérieures n'ont toujours pas résolu, et qui est celui de la lecture à l'écran. La consultation d'un dossier physique, facilement manipulable, classé par sous-thématiques et dans un ordre chronologique est un système très efficace et rapide. Le feuilletage manuel permet à l'œil de sélectionner très rapidement les coupures de presse répondant au sujet recherché. Or, toute informatisation décompose la matière documentaire en unités consultables successivement, d'où une perte de temps et une perte de vision globale du sujet.

Dans les années 1980, il a semblé tout naturel de considérer les nouveaux supports issus des technologies lasers (disque optique numérique), comme le moyen idéal de stocker de gros volumes d'information dans un minimum de place et donc d'associer logique de la documentation informatisée et stockage des documents sous forme d'images. Avec le recul, force est de constater que les différentes expériences de GED menées en matière de documentation de presse sont peu concluantes. Outre les difficultés de consultation à l'écran évoquées plus haut, on peut affirmer sans risque d'être contredit que ces expériences n'ont quasiment pas apporté de gains de productivité. Contrairement à ce que pouvaient prétendre les fabricants de matériel, le temps de préparation des documents n'est pas diminué, c'est même plus souvent le contraire que l'on observe. De plus, dans bien des cas, on a abouti à créer des ateliers de numérisation où officient des aides-documentalistes ou même du personnel moins qualifié. D'autre part, même en considérant les fortes capacités de stockage de ces supports, les volumes sont tels que des problèmes techniques sont assez vite apparus. Sur le plan de l'évolution des technologies, les supports optiques se sont trouvés assez vite rattrapés par les supports optomagnétiques ou magnétiques. Aujourd'hui n'importe quel PC de gamme moyenne possède un disque dur d'au moins $12 \mathrm{GO}$, taille des DON à l'époque. Par ailleurs, la multiplication des supports de stockage suppose l'acquisition de dispositifs mécaniques de type juke-box qui constituent souvent la partie la plus fragile du dispositif, toute panne entraînant des retards considérables dans la chaîne documentaire d'une part, et une impossibilité de servir l'utilisateur, d'autre part.

Peut-être regardera-t-on dans quelques années la GED classique (celle sur support optique) comme une branche avortée de l'évolution technologique. En effet, sur le plan purement technique, elle s'est trouvée en partie dépassée, comme nous l'avons vu par les technologies magnétiques, et sur le plan des usages, par les réseaux. Le développement de l'internet, et notamment du web ouvre de nouvelles perspectives notamment parce qu'il permet de formater les documents selon une 
logique nouvelle, via l'utilisation du langage de balisage HTML. Surtout le web, comme nous le verrons plus loin, modifie la vision que les producteurs d'informations ont de leurs ressources et induisent des changements importants dans les processus de fabrication [COT 98]. L'existence d'une diffusion sur plusieurs supports (papier, bases de données, web, téléphonie mobile), tend à créer un flux transversal de diffusion de l'information, composé d'une matière première qui vient nourrir les principaux supports de diffusion [COT 00]. Certes, aujourd'hui encore, le papier est le produit phare et les systèmes de production ou les logiciels de PAO de type X-Press constituent la base de la production. Pour obtenir des fichiers aptes à nourrir les bases de données, il faut partir des pages montées et les décomposer pour obtenir des fichiers Ascii ou HTML. Néanmoins, cette première étape constitue une rupture importante, car elle signifie que l'on dissocie progressivement la matière informatique qui sert à fabriquer le journal de son support de destination. Dès lors que le contenu brut de l'information est disponible pour un usage multi-support, pourquoi ne pas en faire la matière directe de l'archivage ? Ce qui était déjà en vigueur sur le plan des sources internes, pour nourrir les banques de données de chaque titre, peut être mise en place pour une coopération inter-titres.

C'est l'expérience qui est menée depuis environ deux ans en Angleterre, où les librarians de treize quotidiens nationaux se sont mis d'accord pour s'échanger chaque jour la matière première de leurs publications sous forme de fichiers structurés. A travers cet échange croisé, chacun des titres reçoit l'intégralité des autres titres sous forme électronique et l'intègre à son dispositif documentaire informatisé, quelle qu'en soit la nature (logiciel documentaire, SGBD, etc.). De cette façon, chacun des titres participant au regroupement ${ }^{7}$ dispose en interne de l'intégralité des sources, adaptée à son format de données. C'est évidemment ce type d'entreprise que l'on peut mettre en œuvre aussi en France, qui nous fait dire que la solution de type GED avec archivage optique est en voie de dépassement. En effet, l'échange direct des fichiers, outre le fait qu'il pose moins de problèmes de volume de stockage que l'archivage en mode image, implique de véritables gains de productivité, car une très large partie de la phase de préparation matérielle des documents a disparu.

En ce qui concerne l'expérience anglaise, le papier n'a pas disparu durant la phase de test ; les deux systèmes coexistent donc pour ce qui est de la constitution du stock ${ }^{8}$. En revanche, la réponse aux questions, pour la période récente, se fait le plus souvent en interrogeant la base de données collective et la transmission des articles se fait directement par réseau interne ou par mail. Lorsque la consultation est autorisée directement aux journalistes, ce qui est le cas au Times, on constate une

7. Il est à noter que cette entreprise est purement coopérative, il n'y a pas de chef de file. Elle a émané des responsables de documentation et non des directions des journaux.

8. Ces renseignements m'ont été communiqués par Helen Martin, responsable de la documentation du Guardian, à Londres. 
baisse significative de la consultation du centre de documentation 9 . Il est certain que, de ce point de vue, les pratiques des utilisateurs, les journalistes, ont considérablement évolué en moins de dix ans, et l'impact de l'internet y est pour beaucoup. Dans l'archive de presse, plus peut-être que pour d'autres types de documents, la forme de l'écrit revêt une grande importance. La hiérarchie de l'information se traduit visuellement à travers l'art de la mise en page, et « l'électronification »des archives (du moins dans les banques de données de première génération) possédait, aux yeux du journalistes, ce grave défaut d'écraser complètement cette organisation. D'où une forte réticence, souvent partagée par les documentalistes), à privilégier l'utilisation des banques de données. D'une certaine manière le web a éliminé en partie cet obstacle. Non pas tant parce que les problèmes de lisibilité et de mise en page auraient été résolus ${ }^{10}$, mais parce que le journaliste s'est habitué à consulter des articles à l'écran, ou à faire ses propres recherches sur les moteurs et répertoires du web [GRE 98]. Dans ces conditions, la mise à disposition des « archives » sur les intranets est une tendance importante, qui aura des effets non négligeables sur les pratiques professionnelles et des journalistes, et des documentalistes.

Mais ceci n'est sans doute qu'une première étape. Pratiquement tous les journaux étudient actuellement de près des solutions liées au balisage des documents à travers le langage XML. Contrairement au HTML, le XML permet de conserver au document une structure, et de jouer sur cette structure pour faire communiquer les documents entre eux. D'une certaine manière se fait jour ici la possibilité d'intégrer la logique de la base de données à la production même de la matière journalistique, la structure des articles et donc des documents dans la base étant récurrente : titre, chapeau, sous-titre, texte, signature. Si le standard XML parvient réellement à s'imposer dans le monde de la presse, il est clair que les initiatives d'échange directe de fichiers numériques à la source en serait facilitée, bouleversant totalement l'organisation actuelle des centres de documentation de presse.

De plus, avec XML, le rôle des métadonnées est accru. On peut utiliser les balises, non seulement pour décrire la structure formelle du document (titre, etc.), mais également pour y introduire des éléments de description du contenu, comme par exemple une indexation documentaire. Le numérique a ici cet effet de faire remonter vers l'amont de la chaîne de production les activités documentaires qui se situaient traditionnellement en aval de celle-ci [COT 98]. Les activités de marquage, d'indexation, par exemple, au lieu d'être effectuées a posteriori sur la matière imprimée, le seront sans doute de plus en plus dès la phase de production du journal.

9. Selon Gertrud Erbach, responsable de la documentation du groupe News International (Times, Sunday Times, etc.), le nombre de demandes a diminué de moitié depuis l'installation du système.

10. De nombreux problèmes ergonomiques, liés à la différence de taille de la feuille écrite et de l'écran, subsistent. 
Un autre atout pour le XML est qu'il permet de restituer graphiquement une image de page structurée qui déroute moins le lecteur que le texte en caractères ASCII ${ }^{11}$.

\section{Les archives du web}

La politique des groupes de presse vis-à-vis du web n'a pas toujours été cohérente, les réticences ont été nombreuses, et on peut dire encore à l'heure actuelle que le modèle économique de la diffusion sur l'internet n'est pas évident. Nous n'évoquerons pas cependant ici ces problèmes de stratégie, ni la question des droits d'auteurs qui a compliqué grandement la question ${ }^{12}$. Nous souhaitons surtout soulever ici un problème encore peu évoqué. Il est certain, bien que ce phénomène soit encore peu étudié, que le web bouleverse la logique des médias traditionnels, notamment en bousculant les frontières soigneusement établies depuis un bon siècle : les agences de presse diffusent en fil continu, essentiellement à l'attention des médias auxquels elles fournissent une part de leur matière première; les quotidiens publient chaque jour (parfois en plusieurs éditions successives, mais ce phénomène est moins prégnant aujourd'hui qu'au début du siècle), les hebdomadaires publient chaque semaine, les radios et télevisions diffusent du son et de l'image. Or le réseau est un support de diffusion continu et un support de diffusion multimédia alliant texte, image et son. La recomposition actuelle du paysage médiatique donne ceci :

- les agences de presse, par des accords avec les journaux ou les sites portails diffusent directement au grand public une partie au moins de leurs fils ;

- les hebdomadaires ont vite compris qu'une mise à jour une fois par semaine était incompatible avec la logique du réseau et s'orientent vers une production d'information quotidienne ;

- les quotidiens sont mis à jour plusieurs fois par jour et leur fonctionnement sur le web se rapproche de celui des agences de presse ;

- tous les supports, quelle que soit leur fréquence de diffusion s'orientent de plus en plus vers la création d'une information autonome, spécifique au web, qui n'existe pas sur le support papier. Newsweek par exemple précise sur son site Web exclusive, lorsque les papiers ne sont présents que sur le web. Le site du Figaro distingue les

11. Parallèlement au XML, ou en complémentarité avec celui-ci, l'évolution des formats de type PDF de chez Adobe permet également de restituer des documents qui conservent leur forme originelle et leur mise en page, palliant en partie l'inconvénient soulevé ci-dessus à propos de la lecture de documents électroniques.

12. Il est significatif de voir que la technologie de l'internet a soulevé des interrogations que l'on aurait pu évoquer bien avant, notamment dès la diffusion des archives sur les banques des données des serveurs. Mais la visibilité de ces technologies étant beaucoup plus faible et leur caractère spectaculaire beaucoup moins affirmé, les syndicats de journalistes, entre autres, ne se sont préoccupés de cette question qu'avec l'arrivée du web. 
articles siglés Figaro, qui sont un doublon des articles édités dans le quotidien, et les articles siglés Figaro.fr, qui sont spécifiques au web ;

- les radios ${ }^{13}$ et télévisions diffusent des reportages écrits et des nouvelles, en plus de la mise à disposition de leurs archives sonores ou visuelles ${ }^{14}$.

Les futurs historiens des médias se pencheront certainement sur la dernière décennie du $\mathrm{XX}^{\mathrm{e}}$ siècle avec un intérêt tout particulier. Le chroniqueur spécialisé Steve Outing ${ }^{15}$ n'hésite pas à écrire que les entreprises de presse doivent d'abord se concevoir comme des «news companies » et non comme des journaux, ou radios ou télévisions. Il estime que la fonction de délivrance de l'information peut et doit se manifester sur plusieurs canaux simultanément. Sur le plan de la gestion des archives, cette situation nouvelle nous semble soulever deux problèmes distincts :

- en premier lieu, celui de l'archivage même des pages HTML. La conservation de la mémoire des sites se trouve souvent séparée de celle de la mémoire du journal lui-même, et ce d'autant plus que toutes deux relèvent souvent de services distincts, voire de filiales ;

- en second lieu, cette information originale ne rentre pas (encore) dans les sources externes conservées dans les centres de documentation. Le besoin ne s'en fait pas encore sentir avec évidence et pourtant, si la tendance à développer une information spécifiquement conçue pour le web se confirme, il y aura là une lacune qu'il faudra bien combler.

\section{Conclusion}

Jusqu'à présent, les différents avatars de la documentation informatisée (passage aux banques de données, puis à la GED) n'avaient pas fondamentalement changé la chaîne documentaire en vigueur dans les centres de documentation de presse. Certaines activités se trouvaient transférées sur d'autres supports, mais l'enchaînement des tâches et surtout la philosophie des services rendus ne se trouvaient guère altérées.

La nouvelle génération de technologies qui se trouve appliquée aux archives de presse, comme d'ailleurs dans d'autres secteurs, pourrait bien, cette fois-ci apporter de sérieux bouleversements à la fonction. D'une part, la généralisation de la pratique d'échanges de fichiers amènera un allègement des tâches de traitement physique et

13. Par exemple le service multimédia du groupe Radio-France a embauché des journalistes dits «web-trotteurs », qui collectent l'information et la diffusent sous forme écrite aussi bien que sonore.

14. Nous n'aborderons pas ces supports dans cet article.

15. La chronique «Stop the presses!» est disponible par mail et sur le site www.planetarynews.com 
unifiera l'ensemble des archives en un seul corpus électronique. D'autre part, l'existence des intranets, la pratique croissante des utilisateurs, habitués à consulter l'information en direct sur le web, modifieront les demandes et le travail de recherche effectué par les documentalistes. Certains besoins seront ainsi directement traités, soit par les systèmes automatiques en amont, soit par les utilisateurs en aval. En revanche, de nouveaux besoins apparaîtront, certains rapprochant en partie les services de documentation des services de production: balisage des fichiers, contrôle qualité des bases de données et des sites ; d'autres, rapprochant la fonction documentaire de la fonction journalistique: veille sur des sujets d'actualité, repérage de sources nouvelles, exploitation fine du web, etc.

\section{Bibliographie}

[ALN 84] Albert P., Les banques de données de presse en France, Jean-Cyrille Godefroy, 1984.

[CHA 98] Chabin M.-A., «Patrimoine numérique : révolutions et racines », Document numérique, vol 2. n 3-4, 1998.

[CHA 99] ChabIN M.-A., «Exigences numériques et besoins documentaires », Solaris, décembre 1999-janvier 2000.

[COT 91] CотTE D., Stratégie documentaire dans la presse, ESF, 1991.

[COT 98] COTTE D., « Le texte numérique et l'intériorisation des dispositifs documentaires », Document numérique, vol 2. n 3-4, 1998.

[COT 00] CотTE D., « Représentation des connaissances et convergence numérique, le défi de la complexité », Document numérique, 2000.

[LET 96] LETEINTURIER C., L'identité professionnelle des documentalistes: le cas des médias, ADBS, 1996.

[VAN 99] VANDENDORPE C., Du papyrus à l'hypertexte, La Découverte, 1999. 